\title{
ON TORSION-FREE PERIODIC RINGS
}

\author{
R. R. KHAZAL, S. BREAZ, AND G. CĂLUGĂREANU \\ Received 15 February 2005
}

We characterize several large classes of periodic rings: periodic rings with identity, finiterank torsion-free periodic rings, and rank-two torsion-free periodic rings.

\section{Introduction}

There is a great deal of literature on periodic rings, respectively, torsion-free rings (especially of rank two). The aim of this paper is to provide a link between these two topics.

All groups considered here are Abelian, with addition as the group operation. By order of an element we always mean the additive order of this element. All rings are associative but not necessarily with identity. The additive group of the $\operatorname{ring} R$ will be denoted by $R^{+}$. $M_{n}(R)$ denotes the ring of all the $n \times n$ matrices with entries in $R$.

A ring $R$ is called periodic if for each $x \in R$, the set $\left\{x, x^{2}, x^{3}, \ldots\right\}$ is finite, or equivalently, for each $x \in R$ there are positive integers $m(x), n(x)$ such that $x^{m(x)}=x^{m(x)+n(x)}$. However, periodic rings can also be defined (see [20]) by requiring that (i) the multiplicative semigroup of $R$ is periodic, or, (ii) if $a \in R$, then a power of $a$ generates a finite subring. Examples of periodic rings are finite rings, nil rings, and direct sums of matrix rings over finite fields. $\mathbf{Z}$, the ring of all the integers, is not periodic.

Research on periodic rings (the term "periodic" seems to have been first used by Chacron [16]) was mainly done in two directions:

(i) finding sufficient conditions on periodic rings which imply commutativity, Bell being the prominent name in this direction (all over the last 40 years; e.g., see $[10,11,12])$ but also Abu-Khuzam and Yaqub (see $[1,2,13,26])$, respectively,

(ii) finding structure results for some special classes of periodic rings (e.g., see [3, 5, 12]).

However, it should be noticed that the starting point for these investigations was the Jacobson theorem, whose proof contains many ideas which could be used also in more general contexts.

For later convenience we state here some elementary properties for a periodic ring.

(iii) Any infinite-order element is a zero divisor (in the subring generated by itself).

(iv) Every idempotent in $R$ has finite order.

(v) For each $a \in R$ some power of $a$ is idempotent. 
On the other hand, research on the additive groups of rings begun much earlier. Defining ring structures on Abelian groups was first done by Beaumont [6] who considered rings on direct sums of cyclic groups. Nearly at the same time, Szele investigated nil rings [24] and Beaumont and Zuckerman described the rings on subgroups of the rationals.

Satisfactory results were obtained later by Beaumont and Pierce for finite-(and especially 2) rank torsion-free groups, see [7,8]. Szele began the program of investigating the additive structures of rings by the study of nilpotent rings (see [25]). However, a complete status of the results (previous to 1973) is given in the Fuchs treatise [19]. As of special interest for our paper, we also mention Freedman [18] and Stratton [23] who proved that nonnil torsion-free Abelian groups of rank two possess a unique minimal type, and their typeset has cardinality at most three. Here typeset $(R)$, the typeset of $R$ (or $R^{+}$), denotes the set of all types of the elements in $R$. For the definition of height and type of an element, we refer to [19]. For any group $G$ and any type $\tau, G(\tau)=\{x \in G \mid t(x) \geq \tau\}$. For a torsion-free group $G, E(G)$ denotes the endomorphism ring and $Q E(G)=\mathbf{Q} \otimes_{\mathrm{Z}} E(G)$ the quasiendomorphism ring.

Our main results can be summarized as follows. In Section 2, we determine the structure of the periodic rings with identity. In Section 3, we characterize periodic rings which have a finite-rank torsion-free underlying additive group, obtaining as a by-product a special case confirmation of Köthe's conjecture. In Section 4, we characterize the periodic torsion-free rings of rank two.

\section{Periodic rings with identity}

Given any ring $R$, for any fixed $a \in R$, the left and right multiplications with $a$ are endomorphisms of $R^{+}$. Therefore, fully invariant subgroups of $R^{+}$are necessarily ideals in $R$, no matter how multiplication is defined.

As a special case, the torsion part $T(R)$ is a (two-sided) ideal of $R$. Moreover, the primary components $R_{p}$ ( $p$ prime numbers) of $R^{+}$are also ideals of $R$, and every ring with torsion additive group decomposes (as a ring): $R=\bigoplus_{p \in \mathbf{P}} R_{p}, \mathbf{P}$ denoting the set of all prime numbers. A ring will be called a $p$-ring ( $p$ prime number) if its additive group is an (Abelian) $p$-group. An Abelian group is bounded if there exists a positive integer $n$ such that $n R=\{0\}$.

Definition 2.1. A ring property $\Lambda$ is called non- $Z$, if the ring of integers does not have property $\Lambda$.

Examples of such properties are $\Lambda \equiv$ has zero divisors, or, $\Lambda \equiv$ periodic.

Proposition 2.2. Let $R$ be a ring with identity which satisfies a non-Z property $\Lambda$ together with its subrings. Then $R^{+}$is torsion. Moreover, $R^{+}$is bounded.

Proof. If $1_{R}$ denotes the identity, there is a canonical ring homomorphism $f: \mathbf{Z} \rightarrow R$ such that $f(n)=n 1_{R}$, $\operatorname{ker} f=(\operatorname{char}(R))$, the ideal generated by the characteristics of $R$, and $\operatorname{im} f=\langle 1\rangle \simeq \mathbf{Z} / \operatorname{ker} f$, the subring generated by $1_{R}$. Together with $R,\langle 1\rangle \simeq \mathbf{Z} / \operatorname{ker} f$ has property $\Lambda$ and so, $\operatorname{ker} f=(\operatorname{char}(R)) \neq\{0\}$. Since $\operatorname{char}(R)=\operatorname{ord}_{R^{+}}\left(1_{R}\right)$, it follows that $1_{R} \in T(R)$. Hence $T(R)=R$, the torsion part being an ideal in $R$.

As for the last claim, if $n=\operatorname{char}(R)=\operatorname{ord}_{R^{+}}\left(1_{R}\right)$, for an arbitrary element $r \in R, n r=$ $n\left(1_{R} r\right)=\left(n 1_{R}\right) r=0$, and $n R=\{0\}$. 
Corollary 2.3 (see [19]). A structure of ring with (left) identity exists on a torsion group $G$ if and only if $G$ is bounded.

COROLlaRY 2.4. Every periodic ring with identity is torsion (as a group). Moreover, it is bounded, and so, it is a direct sum of cyclic groups.

As a special case, any semisimple periodic ring $R$ is bounded (this will be used in the next section).

Corollary 2.5. Every periodic ring with identity decomposes (as a ring) in a direct sum of p-rings. Each periodic p-ring is (as a group) a direct sum of cyclic p-groups.

Corollary 2.6 (see [21]). A periodic ring with identity such that $R^{+}$is finitely generated, is finite.

According to Corollary 2.5, the structure of periodic rings with identity reduces to $p$ rings which (as groups) are direct sums of cyclic $p$-groups. A special case of an early result due to László Fuchs settles this.

Theorem 2.7 (see [19]). A multiplication $\mu$ on a direct sum $G=\bigoplus_{i \in I}\left\langle a_{i}\right\rangle$ of cyclic $p$ groups is completely determined by the values $\mu\left(a_{i}, a_{j}\right)$ with $a_{i}, a_{j}$ running over this $p$-basis of $G$. Moreover, any choice of $\mu\left(a_{i}, a_{j}\right) \in G$ with $a_{i}, a_{j}$ from this $p$-basis of $G$-subject to the condition $\operatorname{ord}\left(\mu\left(a_{i}, a_{j}\right)\right) \leq \min \left(\operatorname{ord}\left(a_{i}\right), \operatorname{ord}\left(a_{j}\right)\right)$-extends to a multiplication on $G$. The multiplication is associative (commutative) if (and only if) it is associative (commutative) on the p-basis $\left\{a_{i}\right\}_{i \in I}$.

More can be done (this is the last needed step): $G$ being bounded, any element $a_{i_{0}}$ of maximum order of this $p$-basis can be taken as identity of a ring, by letting $a_{i_{0}}$ act as multiplication by 1 on $\left\langle a_{i_{0}}\right\rangle$ and by trivial multiplication on the other summands (see [19, Theorem 120.8]).

It should be noted that a function $\mu: G \times G \rightarrow G$ is called a multiplication on $G$ if it satisfies

$$
\mu(a, b+c)=\mu(a, b)+\mu(a, c), \quad \mu(a+b, c)=\mu(a, c)+\mu(b, c)
$$

for all $a, b, c$ in $G$. Further, if $G=\bigoplus_{i \in I} H_{i}$ and $H_{i}$ are fully invariant subgroups of $G$, multiplications on $H_{i}(i \in I)$ extend to multiplications on $G$ (and conversely).

According to [19], an Abelian group is called a nil group if there is no ring structure on $G$ other than the zero-ring.

Theorem 2.8 (Szele [24]). A torsion group is nil if and only if it is divisible.

\section{Torsion-free periodic rings of finite rank}

Notice that for an arbitrary ring (denoting by $J(R)$ and $\mathrm{Nil}(R)$ the Jacobson and the nilradicals, resp.) the following statements (known as Köthe's conjecture) are equivalent:

(i) the upper nilradical contains every nil left ideal;

(ii) the sum of two nil left ideals is necessarily nil;

(iii) $\operatorname{Nil}\left(\mathcal{M}_{n}(R)\right)=\mathcal{M}_{n}(\operatorname{Nil}(R))$ for all rings and for all $n$;

(iv) $J(R[\lambda])=\operatorname{Nil}(R)[\lambda]$ for all rings $R$, where $\lambda$ is an indeterminate commuting with all elements of ring. 
From the elementary properties we mentioned in the introduction it follows that any periodic torsion-free ring is nilpotent. Moreover (for an elementary proof see [21]) the following holds.

Lemma 3.1. A torsion-free ring is periodic if and only if it is nil.

COROLlary 3.2. If Köthe's conjecture holds, the matrix ring of a periodic torsion-free ring is also periodic.

Next, recall that if $R$ is a torsion-free ring of finite rank, then $\mathbf{Q} R=\mathbf{Q} \otimes R$ becomes in a natural way a finite-dimensional Q-algebra (this comes back to Cartan and Eilenberg, see [14] or [19, Section 119]). This is a divisible envelope for $R^{+}$, and the dimension of $\mathbf{Q} R$ over $\mathbf{Q}$ equals the rank of $R^{+}$. $\mathbf{Q} R$ may have an identity even if $R$ does not (actually this happens exactly when there is an element $e$ and an integer $n$ such that $e x=n e x$ for all elements $x$ in $R$ ). Using the previous lemma it follows that $R$ is a periodic ring if and only if $\mathbf{Q} R$ is periodic.

The following result shows that in the torsion-free finite rank case, any periodic ring must be nilpotent (the converse obviously also holds).

Theorem 3.3. Let $R$ be a periodic torsion-free ring of rank $n$. Then $R^{n+1}=0$.

Proof. Since $R$ is periodic, every element of $R$ is nilpotent. Thus, the endomorphisms of the group $R^{+}$of the form $t_{r}: R \rightarrow R, t_{r}(x)=r x$, are nilpotent endomorphisms, hence they belong to $N\left(\mathrm{E}\left(R^{+}\right)\right.$), the nil-radical of the endomorphism ring of $R^{+}$. But (see [4, Theorem 9.1]) this nil-radical is nilpotent and so there exists a positive integer $k>0$ such that $t_{r_{1}} \cdots t_{r_{k}}=0$ for any $r_{1}, \ldots, r_{k} \in R$. Therefore $R$ is a nilpotent ring.

Next, if $R$ is a torsion-free ring of finite rank, the finite-dimensional $\mathbf{Q}$-algebra $\mathbf{Q} R=$ $\mathbf{Q} \otimes R$ is an Artinian $\mathbf{Q}$-algebra. As previously noticed, $R$ is a periodic ring if and only if $\mathbf{Q} R$ is periodic (indeed, for all $s \in R, \exists m: r^{m}=0$ implies for all $\alpha s \in \mathbf{Q} R(\alpha \in \mathbf{Q}) \exists m$ : $\left.(\alpha r)^{m}=\alpha^{m} r^{m}=0\right)$.

But $\mathbf{Q} R$ is an $n$-dimensional $\mathbf{Q}$-algebra, hence every strictly descending chain of $\mathbf{Q}$ ideals of $\mathbf{Q} R$ has at most $n$ nonzero terms. Since $\mathbf{Q} R$ is nilpotent as a periodic ring, we use the chain $(\mathbf{Q} R) \geq(\mathbf{Q} R)^{2} \geq \cdots \geq(\mathbf{Q} R)^{n+1}$, and the fact that if $(\mathbf{Q} R)^{s}=(\mathbf{Q} R)^{s+1}$, then $(\mathbf{Q} R)^{s}=(\mathbf{Q} R)^{k}$ for all $k>s$, to obtain $0=(\mathbf{Q} R)^{n+1}=\mathbf{Q} R^{n+1}$.

COROLLARY 3.4. Let $R$ be a torsion-free ring of finite rank. Then $R$ is periodic if and only if $R$ is nilpotent.

In the literature, rings which are finitely generated as rings have been rarely studied. Obviously, if a ring is finitely generated as a group, it is also finitely generated as a ring.

COROLlaRY 3.5. Let $R$ be a periodic ring of finite torsion-free rank. Then it is finitely generated as a ring if and only if it is finitely generated as a group.

Proof. Let $n$ be the rank of $R^{+}$. If $R=\left\langle r_{1}, \ldots, r_{m}\right\rangle$, then

$$
R^{+}=\left\langle\prod_{i=1}^{k} x_{i} \mid k=1, \ldots, n, x_{i} \in\left\{r_{1}, \ldots, r_{m}\right\}\right\rangle .
$$


Remark 3.6. Actually more can be proved (see [21]):

(A) if $R$ is a commutative periodic ring, the two ways of being finitely generated are equivalent.

COROLlaRy 3.7. If $R$ is a periodic finite-rank torsion-free ring, then $M_{n}(R)$ is periodic.

COROLlARY 3.8. If $R$ is a rank 1 torsion-free periodic ring, then $R^{2}=0$.

\section{Rank-two torsion-free periodic rings}

In this section, $R$ denotes a torsion-free ring of rank two. We continue the discussion initiated by Beaumont and Wisner in [9] and continued by Beaumont and Pierce in [8].

The structure of rank-two torsion-free groups which admit a nontrivial noncommutative multiplication was intensively investigated in $[9,17]$. First we show that such periodic rings are commutative. We recall that there exists (up to an isomorphism) only one structure of two-dimensional nilpotent Q-algebra (see [15] or [8, Section 9]) and this is commutative. Using this and the $\mathbf{Q}$-algebra $\mathbf{Q} R$, we obtain the following.

Proposition 4.1. A rank-two torsion-free periodic ring is commutative.

An important result towards finding the structure of the not nil rank-two torsion-free rings was a theorem due to Freedman and Stratton (see [18, 23]):

(A) the typeset of a not nil rank two torsion-free ring possesses a unique minimal element, and has at most three elements.

The next result determines rank-two torsion-free groups which admit a nontrivial multiplication of periodic type.

THEOREM 4.2. Let $G$ be a rank-two torsion-free group. $G$ admits a nontrivial multiplication of periodic type if and only if there exists a proper pure subgroup $H$ of $G$ such that (type $(G / H))^{2} \leq$ type $(H)$.

Proof. Let $R$ be a periodic ring such that $R^{2} \neq 0$ and the additive group $R^{+}$is isomorphic to $G$. Then there exists $r \in R$ such that the (left) multiplication with $r$ (i.e., $t_{r}$ : $\left.R \rightarrow R, t_{r}(x)=r x\right)$ is a nonzero endomorphism of $R^{+}$. Since $R^{3}=0$, we obtain $t_{r}^{2}=0$ so that $H=\operatorname{ker}\left(t_{r}\right)$ is a pure subgroup of $R$ with $t_{r}(R) \leq H$. Therefore there is a nonzero monomorphism $R / H \rightarrow H$. Moreover, since $H$ is of rank 1 , for every $h \in H$ there is a rational number $q$ and $x \in R$ such that $h=q r x$. Consequently $R H=0$ and it follows that $r \notin H$. If $x_{1}, x_{2} \in R$, there are integers $m_{i}, n_{i}$, and elements $h_{i} \in H(i \in\{1,2\})$ such that $n_{i} x_{i}=m_{i} r+h_{i}$. Hence $n_{1} n_{2} x_{1} x_{2}=m_{1} m_{2} r^{2} \in H$ and so $r^{2} \neq 0$ and $(\operatorname{type}(G / H))^{2} \leq$ type $(H)$.

Conversely, suppose $H$ is a proper subgroup of $G$ with (type $(G / H))^{2} \leq \operatorname{type}(H)$. Let $S \leq T$ be rational groups such that $1 \in S$ with type $(G / H)=\operatorname{type}(S)$ and type $(H)$ $=$ type $(T)$. From the type hypothesis, we can suppose $S^{2}=\left\{s_{1} s_{2} \mid s_{1}, s_{2} \in S\right\} \subseteq T$. Fix $a \in G$ such that $S(a+H)=G / H$ and $h \in H$ with $T h=H$ and define a multiplication as follows: if $x_{1}, x_{2} \in G$ and $n_{i} x_{i}=m_{i} a+h_{i}$ with $m_{i} / n_{i} \in S$ and $h_{i} \in H$ for all $i \in\{1,2\}$, then $x_{1} x_{2}=\left(m_{1} m_{2} / n_{1} n_{2}\right) h$. It is easy to verify that this multiplication defines a periodic ring structure on $G$. 
Remark 4.3. From the previous proof, notice that if $G$ is a rank $n$ torsion-free group which admits a nontrivial periodic ring multiplication, then $G$ has a nonzero nilpotent endomorphism. Hence, in the $n=2$ case, using [22, Theorem 7.1], the quasiendomorphism ring of $G$ must be one of the following matrix rings:

(i) $M_{2}(\mathbf{Q})$, or

(ii) the ring of all $2 \times 2$ rational triangular matrices, or

(iii) the ring of all $2 \times 2$ rational triangular matrices with equal diagonal entries.

We summarize from [4, Section 3] what we need in the sequel. For a torsion-free group $G$ of rank two, we have the following possible situations:

(a) the quasiendomorphism ring of $G$ is isomorphic to $M_{2}(\mathbf{Q})$ if and only if $G=H \oplus$ $K$ with type $(H)=\operatorname{type}(K)$ (i.e., $G$ is homogeneous completely decomposable),

(b) the quasiendomorphism ring of $G$ is isomorphic to the ring of all $2 \times 2$ rational triangular matrices if and only if $G=H \oplus K$ with type $(H)<\operatorname{type}(K)$,

(c) the quasiendomorphism ring of $G$ is isomorphic to the ring of all $2 \times 2$ rational triangular matrices with equal diagonal entries if and only if $G$ is strongly indecomposable, $|\operatorname{typeset}(G)|=2$, and $G$ has a nilpotent endomorphism.

Notice that in all these cases typeset $(A)=\left\{\tau_{1}, \tau_{2}\right\}$ with $\tau_{1} \leq \tau_{2}$.

Here, a torsion-free group $G$ is strongly indecomposable if whenever $0 \neq k \in \mathbf{Z}$ and $k G \subseteq H \oplus K \subseteq G$, then $H=0$ or $K=0$.

TheOREM 4.4. A rank-two torsion-free group $G$ admits a nontrivial periodic ring structure if and only if one of the following conditions holds:

(i) $G$ is homogeneous completely decomposable of idempotent type, or

(ii) $G=H \oplus K$ with type $(H)^{2}<$ type $(K)$, or

(iii) $G$ is strongly indecomposable, typeset $(G)=\left\{\tau_{1}, \tau_{2}\right\}$ with $\tau_{1}<\tau_{2}$, and type $\left(G / G\left(\tau_{2}\right)\right)^{2}$ $\leq \tau_{2}$.

Proof. The (i) case corresponds to (a) in the preceding discussion. In this situation every pure subgroup is a direct summand, hence the kernel $\operatorname{ker}(f)$, for every nilpotent endomorphism $f$ of $G$, is a direct summand too. Then type $(G / \operatorname{ker}(f))=\operatorname{type}(\operatorname{ker}(f))=$ type $(G)$ and so type $(G)$ is idempotent. The same conclusion can be deduced from [23].

If $G$ satisfies one of the conditions (b) or (c), the typeset $(G)=\left\{\tau_{1}, \tau_{2}\right\}$ with $\tau_{1}<\tau_{2}$. If $f$ is a nonzero nilpotent endomorphism of $G$, then $\operatorname{ker}(f)=G\left(\tau_{2}\right)$ (see $[4$, Section 3]). The proof is now complete using Theorem 4.2.

\section{References}

[1] H. Abu-Khuzam, A commutativity theorem for periodic rings, Math. Japon. 32 (1987), no. 1, $1-3$.

[2] Commutativity results for periodic rings, Acta Math. Hungar. 58 (1991), no. 3-4, 273277.

[3] E. P. Armendariz, On infinite periodic rings, Math. Scand. 59 (1986), no. 1, 5-8.

[4] D. M. Arnold, Finite Rank Torsion Free Abelian Groups and Rings, Lecture Notes in Mathematics, vol. 931, Springer, New York, 1982.

[5] M. Ashraf, Structure of certain periodic rings and near rings, Rend. Sem. Mat. Univ. Politec. Torino 53 (1995), no. 1, 61-67. 
[6] R. A. Beaumont, Rings with additive group which is the direct sum of cyclic groups, Duke Math. J. 15 (1948), no. 2, 367-369.

[7] R. A. Beaumont and R. S. Pierce, Torsion free groups of rank two, Mem. Amer. Math. Soc. 38 (1961), 1-41.

[8]_ Torsion-free rings, Illinois J. Math. 5 (1961), 61-98.

[9] R. A. Beaumont and R. J. Wisner, Rings with additive group which is a torsion-free group of rank two, Acta Sci. Math. (Szeged) 20 (1959), 105-116.

[10] H. E. Bell, Some commutativity results for periodic rings, Acta Math. Acad. Sci. Hungar. 28 (1976), no. 3-4, 279-283.

[11] On commutativity and structure of certain periodic rings, Glas. Mat. Ser. III 25(45) (1990), no. 2, 269-273.

[12] H. E. Bell and S. Ligh, Some decomposition theorems for periodic rings and near-rings, Math. J. Okayama Univ. 31 (1989), no. 1, 93-99.

[13] H. E. Bell and A. Yaqub, Generalized periodic rings, Int. J. Math. Math. Sci. 19 (1996), no. 1, 87-92.

[14] H. Cartan and S. Eilenberg, Homological Algebra, Princeton University Press, New Jersey, 1956.

[15] A. Cayley, On double algebra, Proc. London Math. Soc. 15 (1884), 185-197.

[16] M. Chacron, On quasi periodic rings, J. Algebra 12 (1969), 49-60.

[17] S. Feigelstock, Non-commutative rank two torsion free rings, Comment. Math. Univ. St. Paul. 32 (1973), 51-53.

[18] H. Freedman, On the additive group of a torsion-free ring of rank two, Publ. Math. Debrecen 20 (1973), 85-87.

[19] L. Fuchs, Infinite Abelian Groups. Vol. II, Pure and Applied Mathematics. Vol. 36-II, Academic Press, New York, 1973.

[20] R. R. Khazal, Multiplicative periodicity in rings, Acta Sci. Math. (Szeged) 41 (1979), no. 1-2, 133-136.

[21] R. R. Khazal and S. Dăscălescu, Periodic rings with finitely generated underlying group, Int. J. Math. Math. Sci. 2004 (2004), no. 36, 1887-1892.

[22] J. D. Reid, On the ring of quasi-endomorphisms of a torsion-free group, Topics in Abelian Groups (Proc. Sympos., New Mexico State University, 1962), Scott, Foresman and Co., Illinois, 1963, pp. 51-68.

[23] A. E. Stratton, The type set of torsion-free rings of finite rank, Comment. Math. Univ. St. Paul. 27 (1979), no. 2, 199-211.

[24] T. Szele, Zur Theorie der Zeroringe, Math. Ann. 121 (1949), 242-246 (German).

[25] _ Nilpotent Artinian rings, Publ. Math. Debrecen 4 (1955), 71-78.

[26] A. Yaqub, Structure of weakly periodic rings with potent extended commutators, Int. J. Math. Math. Sci. 25 (2001), no. 5, 299-304.

R. R. Khazal: Department of Mathematics and Computer Science, Faculty of Science, Kuwait University, P.O. Box 5969, Safat 13060, Kuwait

E-mail address: khazal@sci.kuniv.edu.kw

S. Breaz: Faculty of Mathematics and Computer Science, "Babes-Bolyai" University, 1 Kogalniceanu Street, 400084 Cluj-Napoca, Romania

E-mail address: bodo@math.ubbcluj.ro

G. Călugăreanu: Department of Mathematics and Computer Science, Faculty of Science, Kuwait University, P.O. Box 5969, Safat 13060, Kuwait

E-mail address: calu@sci.kuniv.edu.kw 


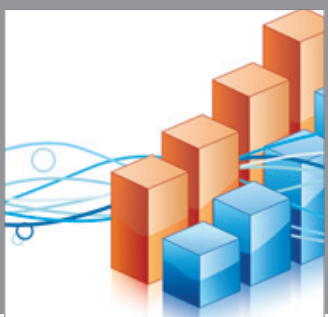

Advances in

Operations Research

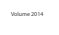

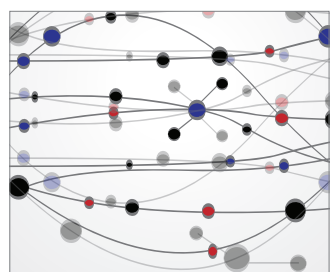

\section{The Scientific} World Journal
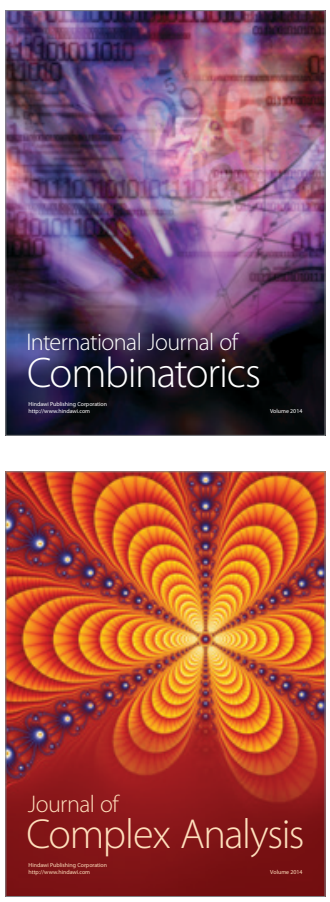

International Journal of

Mathematics and

Mathematical

Sciences
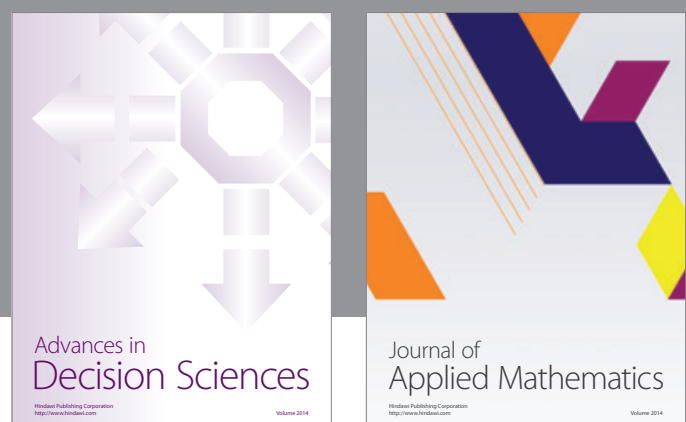

Journal of

Applied Mathematics
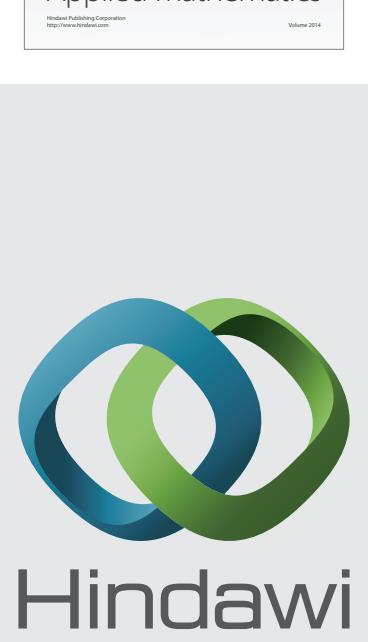

Submit your manuscripts at http://www.hindawi.com
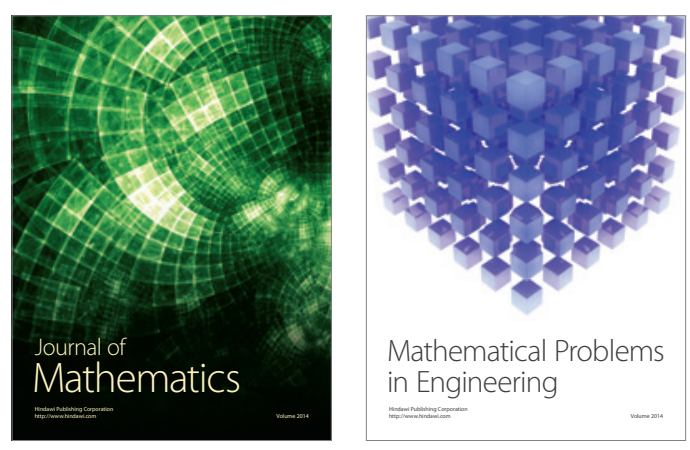

Mathematical Problems in Engineering
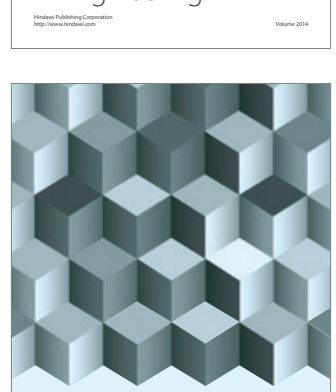

Journal of

Function Spaces
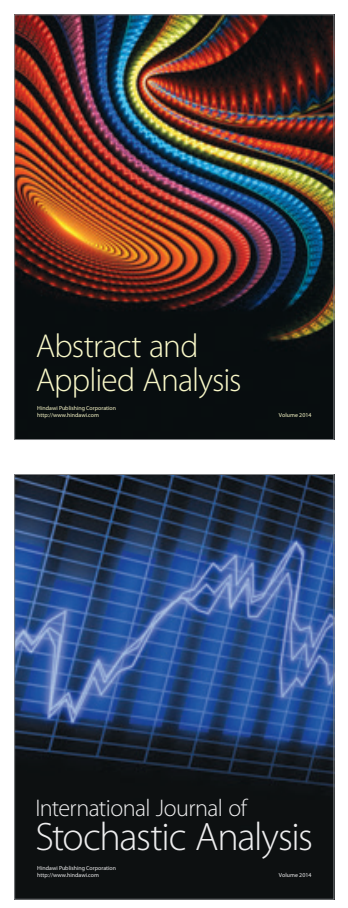

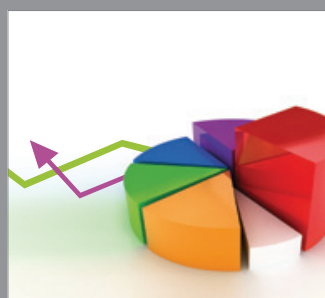

ournal of

Probability and Statistics

Promensencen
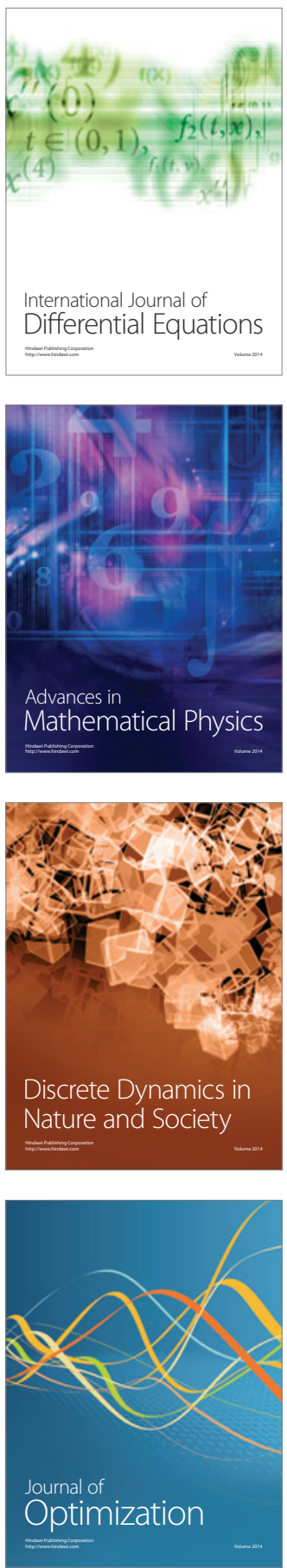